Trauma Surgery \& Acute Care Open

\title{
Minor change in initial PEEP setting decreases rates of ventilator-associated events in mechanically ventilated trauma patients
}

\author{
Ethan Ferrel, ${ }^{1}$ Kristina M Chapple (D) , ' Liviu Gabriel Calugaru, ${ }^{2}$ Jennifer Maxwell, ${ }^{2}$ \\ Jessica A Johnson (D) ,' 2 Andrew W Mezher (D) ,' James N Bogert (D) ,' Hahn Soe-Lin,' \\ Jordan A Weinberg ${ }^{2}$
}

${ }^{1}$ Creighton University School of Medicine Phoenix Regional Campus, Phoenix, Arizona, USA 2Department of Surgery, Dignity Health/St. Joseph's Hospital and Medical Center, Phoenix, Arizona, USA

\section{Correspondence to} Dr Jordan A Weinberg; Jordan. Weinberg@dignityhealth.org

Presented at the 78th Annual Meeting of the American Association for the Surgery of Trauma, September 18-21, 2019 in Dallas, Texas.

Received 13 February 2020 Revised 22 April 2020 Accepted 22 April 2020 (c) Author(s) (or their employer(s)) 2020. Re-use permitted under CC BY-NC. No commercial re-use. See rights and permissions. Published by BMJ.

\section{To cite: Ferrel $\mathrm{E}$}

Chapple KM, Calugaru LG,

et al. Trauma Surg Acute Care Open 2020;5:e000455.

\begin{abstract}
Background Surveillance of ventilator-associated events (VAEs) as defined by the National Healthcare Safety Network (NHSN) is performed at many US trauma centers and considered a measure of healthcare quality. The surveillance algorithm relies in part on increases in positive end-expiratory pressure (PEEP) to identify VAEs. The purpose of this cohort study was to evaluate the effect of initiating mechanically ventilated trauma patients at marginally higher PEEP on incidence of VAEs. Methods Analysis of level- 1 trauma center patients mechanically ventilated 2+ days from 2017 to 2018 was performed after an institutional ventilation protocol increased initial PEEP setting from 5 (2017) to 6 (2018) $\mathrm{cm} \mathrm{H} \mathrm{H}_{2} \mathrm{O}$. Incidence of VAEs per 1000 vent days was compared between PEEP groups. Logistic regression modelling was performed to evaluate the impact of the PEEP setting change adjusted to account for age, ventilator days, injury mechanism and injury severity. Results 519 patients met study criteria (274 PEEP 5 and 245 PEEP 6). Rates of VAEs were significantly reduced among patients with initial PEEP 5 versus 6 (14.61 per 1000 vent days vs. 7.13 per 1000 vent days; $\mathrm{p}=0.039$ ). Logistic regression demonstrated that initial PEEP 6 was associated with $62 \%$ reduction in VAEs. Conclusions Our data suggest that an incrementally increased baseline PEEP setting was associated with a significantly decreased incidence of VAEs among trauma patients. This minor change in practice may have a major impact on a trauma center's quality metrics. Level of evidence IV.
\end{abstract}

\section{INTRODUCTION}

Mechanical ventilation remains a mainstay of therapy in the care of the critically ill. Patients undergoing mechanical ventilation are susceptible to a wide range of associated conditions. Great efforts continue to be made to identify, stratify, and optimize prevention of the complications of this essential therapy. The most reported complication of mechanical ventilation to date has been ventilator-associated pneumonia (VAP). Relatively subjective elements of the VAP definition along with practice variability with respect to VAP diagnosis have resulted in VAP being a suboptimal quality indicator with respect to benchmarking and quality improvement. ${ }^{1}$
In 2013, the Centers for Disease Control and Prevention (CDC) adopted the National Healthcare Safety Network's (NHSN) definition of ventilator-associated events (VAEs) in an effort to facilitate the objective identification of nosocomial respiratory conditions developed by intubated patients and to simplify surveillance across institutions. The 2013 updates use an algorithm of diagnostic criteria to identify these processes, starting with ventilator-associated conditions (VACs), progressing to infection-related ventilator-associated complications (IVACs), and finally possible VAP (PVAP), all of which are considered VAEs. ${ }^{2}$

Many US institutions report rates of VAEs as a hospital quality measure. Per the VAE algorithm, a patient first requires a baseline period of stability, defined as two or more days of stable or decreasing daily fractional inspired oxygen $\left(\mathrm{FiO}_{2}\right)$ or positive end-expiratory pressure (PEEP) values (figure 1). ${ }^{2}$ Development of a VAE is then defined by an increase in daily minimum $\mathrm{FiO}_{2}$ of 20 points or more over the $\mathrm{FiO}_{2}$ on the first day of the baseline period for two or more consecutive days, and/or an increase in PEEP by $3 \mathrm{~cm} \mathrm{H}_{2} \mathrm{O}$ or more over the first baseline day's minimum PEEP similarly for two or more consecutive days (figure 2).

Once these criteria are met, the patient is defined as having experienced a VAE, triggering a reportable quality metric. ${ }^{2}$ In our institution, an internal review of VAE cases demonstrated that a number of VAEs were triggered by a PEEP increase from $5 \mathrm{~cm} \mathrm{H}_{2} \mathrm{O}$ to $8 \mathrm{~cm} \mathrm{H}_{2} \mathrm{O}$ after 48 hours of mechanical ventilation, unaccompanied by any significant increase in $\mathrm{FiO}_{2}$ (that would have also triggered a VAE). We speculated that this PEEP increase, although meeting metric criteria for a VAE, frequently lacked clinical significance. We further speculated that a change in routine ventilator practice whereby initial PEEP was set at 6 rather than $5 \mathrm{~cm} \mathrm{H}_{2} \mathrm{O}$ might decrease VAE rates simply by excluding these clinically insignificant events. In 2017, this practice change was implemented for intubated trauma patients. The aim of this study was to identify the impact of a new ventilator protocol on the subsequent reporting of VAEs in our intubated trauma patient population. We hypothesized that this slight increase in default initial PEEP would reduce the incidence of VAEs among intubated trauma patients. 
Patient has a baseline period of stability or improvement on the ventilator, defined by $\geq 2$ calendar days of stable or decreasing daily minimum ${ }^{*} \mathrm{FiO}_{2}$ or PEEP values. The baseline period is defined as the 2 calendar days immediately preceding the first day of increased daily minimum $\mathrm{PEEP}$ or $\mathrm{FiO}_{2}$.

*Daily minimum defined by the lowest value of $\mathrm{FiO}_{2}$ or PEEP during the calendar day that is maintained for $>1$ hour

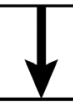

After a period of stability or improvement on the ventilator, VAE is satisfied by the patient having at least one of the following indicators of worsening oxygenation:

1. Increase in daily minimum ${ }^{*} \mathrm{FiO}_{2} \geq 20$ (20 points) over the daily minimum $\mathrm{FiO}_{2}$ of the first day in the baseline period, sustained for $\geq 2$ calendar days.

2. Increase in daily minimum* PEEP values of $>3 \mathrm{cmH}_{2} \mathrm{O}$ over the daily minimum PEEP of the first day on the baseline period $^{\dagger}$, sustained for $\geq 2$ calendar days.

*Daily minimum defined by the lowest value of $\mathrm{FiO}_{2}$ or PEEP during a calendar day that is maintained $>1$ hour.

tDaily minimum PEEP values of $0-5 \mathrm{cmH}_{2} \mathrm{O}$ are considered equivalent for the purposes of VAE surveillance.

Figure 1 Ventilator-associated events surveillance algorithm (adapted from Centers for Disease Control and Prevention (CDC) 2019²).

Materials are developed by CDC and free to use via the CDC website. Reference to specific commercial products, manufacturers, companies, or trademarks does not constitute its endorsement or recommendation by the US government, Department of Health and Human Services, or CDC. $\mathrm{FiO}_{2}$, fractional inspired oxygen; PEEP, positive end-expiratory pressure.

\section{METHODS}

Patients

Patients were identified from the trauma patient registry of St. Joseph's Hospital and Medical Center, an American College of Surgeons-verified level 1 trauma center in Phoenix, Arizona. This retrospective study included trauma patients admitted to the intensive care unit (ICU) between July 1, 2016 and June 30, 2018 who underwent mechanical ventilation for two or more days.

\section{Ventilator management}

Prior to July 1, 2017, standard initial ventilator settings for trauma patients included PEEP setting of $5 \mathrm{~cm} \mathrm{H}_{2} \mathrm{O}$. Commencing July 1, 2017, the initial PEEP setting was changed to $6 \mathrm{~cm} \mathrm{H}_{2} \mathrm{O}$. The section pertaining to initial ventilator parameters excerpted from our Adult Ventilator Management hospital guideline is demonstrated in figure 1 . For the purpose of this study, patients were stratified according to initial PEEP setting by date of admission: fiscal year (FY) 2017 (July 1, 2016 to June 30, 2017) versus FY 2018 (July 1, 2017 to June 30, 2018). No other practice changes were made regarding ventilator management between time periods or at any other time during the study period.

\section{Outcomes}

VAEs were determined by the Department of Quality Management at St. Joseph's Hospital and Medical Center in accordance with the Center for Disease Control NHSN guidelines (figure 1). All patient VAEs over the period of study were provided by the Quality Management team to the study investigators for cross referencing with the trauma registry; thereby all trauma patients with VAEs were identified for this study.
VAP was determined by the performance improvement committee of the Division of Trauma at St. Joseph's Hospital, according to the criteria described in the National Trauma Data Standard Data Dictionary. The clinical practice among the trauma surgeons was to follow an institutional guideline founded on quantitative culture from bronchoalveolar lavage (BAL) effluent. Per our guideline, BAL specimen is obtained for the presence of at least three of four clinical findings: abnormal temperature $\left(>38^{\circ} \mathrm{C}\right.$ or $<36^{\circ} \mathrm{C}$ ), leukocytosis (white cell count $>10 \times 10^{9} / \mathrm{L}$ ) or leukopenia (white cell count $<4 \times 10^{9} / \mathrm{L}$ ), purulent sputum, and new or worsening infiltrate on chest X-ray. Quantitative BAL cultures with equal to or greater than $10^{5} \mathrm{CFU} /$ $\mathrm{mL}$ were considered positive for VAP. All VAPs were reviewed by the performance improvement committee and adjudicated according to the National Trauma Data Standard criteria. Positive cases were logged as such in the institutional trauma registry. Additional outcome variables including discharge disposition and ventilator days were abstracted from the trauma registry.

\section{Statistical analysis}

Cohort characteristics for patients ventilated two or more days were compared between FY 2017 and FY 2018 using a t-test for age, Mann-Whitney $U$ tests for injury severity and number of ventilated days, and a Pearson $\chi^{2}$ test for the proportion of penetrating cases. Means are reported \pm SD and medians with the 25 th to 75 th percentiles. The number of ventilated days was summed to obtain the total ventilated days for trauma patients by year. Mortality and the incidence of VAEs per 1000 patient ventilated days were computed for FY 2017 and FY 2018 and compared using MedCalc's proportions and rate comparison calculators V.18.10 (MedCalc Software bvba, Ostend Belgium; http://wwwmedcalc.org; 2018). The Bonferroni adjustment was applied for post-hoc comparisons. Logistic regression modelling was performed to account for age, ventilator days, penetrating versus blunt injury, and injury severity using SPSS V.25. P values less than 0.05 were considered statistically significant.

\section{RESULTS}

Our institution registered 4037 trauma patients in FY 2017 and FY 2018 combined. Of these, 519 were ventilated for two or more days and therefore met inclusion criteria for our study (274 (52.8\%) patients in FY 2017 and 245 (47.2\%) in FY 2018). Our patient population was $77.8 \%(n=404)$ male with an average age of $43.8 \pm 19.3$ years. Analyses summarized in table 1 were performed to assess for differences in patient and injury characteristics. There were not significant differences in patient age $(\mathrm{p}=0.950)$, injury severity $(\mathrm{p}=0.352)$, ventilated days $(p=0.673)$, proportion of penetrating injuries $(p=0.732)$ or discharge disposition $(\mathrm{p}=0.985)$ between the two groups.

Comparison of rates of VAE between the FY 2017 and FY 2018 groups is demonstrated in table 2 . Twenty-eight patients (10.2\%) in FY 2017 versus 11 patients (4.5\%) in FY 2018 developed VAE $(p=0.014)$. Comparison of VAEs per 1000 ventilator days demonstrated similar results. The rate of VAE per 1000 ventilator days was 14.61 in FY 2017 versus 7.13 in FY $2018(\mathrm{p}=0.039)$.

To evaluate the impact of the practice change with respect to initial PEEP setting, patient characteristics were added as covariates in a multivariate logistic regression model (table 3). Adjusted for patient age, injury severity, mechanism of injury, and ventilator days of 7 days or more, the increase from initial PEEP of 5 to 6 (as represented by FY cohort) was associated with a significant decrease in VAEs (OR=0.38 (95\% CI 0.17 to 0.84$)$ ). 
A Volume ventilation (VV) modes are generally used for the majority of patients, but Pressure Ventilation modes should be considered if peak pressures rise over $40 \mathrm{cmH}_{2} \mathrm{O}$ or if plateau pressures (Pplateau) rise over $30 \mathrm{cmH}_{2} \mathrm{O}$. The initial $\mathrm{WV}$ settings (SIMV, PRVC, or A/C) should be determined based upon the patient's ideal body weight (IBW) and immediate clinical needs.

B Tidal volume (VT) initial setting of 6-8 mL/kg IBW while maintaining Pplateau $<30 \mathrm{cmH}_{2} \mathrm{O}$ and delta $\mathrm{P}<20 \mathrm{cmH}_{2} \mathrm{O}$. Necessary adjustments may range from $4-12 \mathrm{~mL} / \mathrm{kg} \mathrm{IBW}$ to maintain the parameters of Pplateau $<30 \mathrm{cmH}_{2} \mathrm{O}$ and delta $\mathrm{P}<20 \mathrm{cmH}_{2} \mathrm{O}$. Consult physician if unable to maintain these parameters. Calculate IBW.

1. Male IBW $(\mathrm{kg})=50+2.3$ [height (inches) -60 ]

2. Female IBW $(\mathrm{kg})=45.5+2.3$ [height (inches) -60$]$.

C Rate (f): 8-26 breaths/minute adjusted to achieve I:E ratio and maintain desired MV, while maintaining Pplateau $<30 \mathrm{cmH}_{2} \mathrm{O}$ and delta $\mathrm{P}<20 \mathrm{cmH}_{2} \mathrm{O}$.

D $\mathrm{FIO}_{2}$ : Initial setting of 0.6-1.0 (may be less $0.4-1.0$ for postanesthesia recovery) until ABG results are obtained.

1. Initial $A B G$ should be obtained $20-30$ minutes from start of ventilation

2. Pulse oximetry $\left(\mathrm{SpO}_{2}\right)$, and end-tidal $\mathrm{CO}_{2}\left(\mathrm{ETCO}_{2}\right.$ optional), should be correlated with initial $\mathrm{ABG}$

3. Once $A B G s$ are stabilized continue subsequent patient monitoring with continuous pulse oximetry to maintain $\mathrm{SpO}_{2}-$ desired saturation for patient's category.

4. Once $A B G s$ are stabilized, continue subsequent patient monitoring with $\mathrm{ETCO}_{2}$ to maintain patient's normal or within the normal ETCO $\mathrm{C}_{2}$ range (optional).

5. PEEP: Set initial PEEP at $6 \mathrm{cmH}_{2} \mathrm{O}$, unless otherwise indicated. Higher PEEP levels may be required with acute lung injury or acute respiratory distress syndrome.

E Pressure support (PS): Set initial PS at 5-10 $\mathrm{cmH}_{2} \mathrm{O}$, adjusted to reduce work of breathing, patient fatigue, and still support effective ventilation.

F I:E ratio: Adjust to achieve an I:E ratio greater than 1:1 (for example, 1:3). The I:E ratio should be optimized to provide optimum mean airway pressure, lung filling, lung emptying (minimizing air trapping/auto-PEEP), and patient/ventilator synchrony.

Figure 2 Initial ventilator parameters guideline, St. Joseph's Hospital and Medical Center, Phoenix, Arizona. ABG, arterial blood gas; A/C, asssit control; $\mathrm{FiO}_{2}$, fractional inspired oxygen; I:E, inpiration to expiration ratio; MV, minute ventilation; PEEP, positive end-expiratory pressure; PRVC, pressure-regulated volume control; ; SIMV, synchronized intermittent mandatory ventilation .

Mortality associated with VAEs increased from 23.8\% in FY 2017 to $55.6 \%$ in FY 2018 ( $\mathrm{p}=0.330$ ). Additional multivariate logistic regression models were created to evaluate the association between mortality and VAEs for both FY cohorts (table 4). Models were adjusted for age, injury severity and penetrating versus blunt injury. VAEs in FY 2017 did not predict mortality

\begin{tabular}{|c|c|c|c|}
\hline & FY 2017 & FY 2018 & $P$ value \\
\hline Patients (n) & 274 & 245 & - \\
\hline $\begin{array}{l}\text { Patient age years, } \\
\text { mean } \pm S D\end{array}$ & $43.7 \pm 19.4$ & $43.8 \pm 19.1$ & 0.950 \\
\hline Injury Severity Score & $20.0(12.0-27.0)$ & $17.5(10.0-29.0)$ & 0.352 \\
\hline $\begin{array}{l}\text { Ventilated days, median } \\
\text { (25th-75th percentile) }\end{array}$ & $3.0(3.0-9.0)$ & $3.0(2.0-8.0)$ & 0.673 \\
\hline $\begin{array}{l}\text { Penetrating injury } \\
\text { (vs. blunt) }\end{array}$ & $57(20.8 \%)$ & $48(19.6 \%)$ & 0.732 \\
\hline Disposition & & & 0.985 \\
\hline Home & $86(31.4 \%)$ & $78(31.8 \%)$ & \\
\hline $\begin{array}{l}\text { Rehab/skilled nursing } \\
\text { facility }\end{array}$ & $110(40.1 \%)$ & $101(41.2 \%)$ & \\
\hline Died & $59(21.5 \%)$ & $50(20.4 \%)$ & \\
\hline Other & $19(6.9 \%)$ & $16(6.5 \%)$ & \\
\hline
\end{tabular}

FY, fiscal year; Rehab, Rehabilitation.
$(\mathrm{OR}=0.3,95 \% \mathrm{CI}(0.08$ to 1.1$))$. In the FY 2018 cohort, however, VAEs were significantly associated with mortality $(\mathrm{OR}=8.77,95 \% \mathrm{CI}(2.22$ to 34.61$))$.

There were 18 VAP events $(6.6 \%)$ in FY 2017, compared with 12 VAP events $(4.9 \%)$ in FY $2018(\mathrm{p}=0.409)$. The rate per 1000 ventilator days per FY was also similar (9.39 vs. 7.78, $\mathrm{p}=0.614)$. Notably, in FY 2017, among the 18 VAP events, seven had concomitant VAEs. In FY18, among the 12 VAP events, two had concomitant VAEs. From FY 2017 to FY 2018, mortality associated with VAP decreased from $18.2 \%$ to $10.0 \%(p=0.878)$.

\section{DISCUSSION}

The NHSN's development of the VAE algorithm in 2013 was intended to standardize the identification of both non-infectious and infectious nosocomial respiratory conditions in intubated patients. Substantial variability in VAE incidence has been reported, revealing both the need to further investigate the

\begin{tabular}{|c|c|c|c|}
\hline & FY 2017 & FY 2018 & $P$ value \\
\hline \multicolumn{4}{|l|}{ VAES } \\
\hline Patients (n) & 28 & 11 & 0.014 \\
\hline Rate per 1000 ventilator days & 14.61 & 7.13 & 0.039 \\
\hline
\end{tabular}

FY, fiscal year; VAEs, ventilator-associated events. 
Table 3 Summary of logistic regression model predicting VAE

\begin{tabular}{lccc}
\hline & P value & OR & 95\% Cl for OR \\
\hline Age & 0.274 & 0.99 & 0.97 to 1.01 \\
ISS & 0.029 & 1.03 & 1.00 to 1.06 \\
Penetrating injury & 0.619 & 1.30 & 0.46 to 3.67 \\
Vented 7+days & $<0.001$ & 97.47 & 13.08 to 726.65 \\
FY 2018 (Initial PEEP 6) & 0.017 & 0.38 & 0.17 to 0.84 \\
\hline
\end{tabular}

FY, fiscal year; ISS, Injury Severity Score; PEEP, positive end-expiratory pressure; VAE, ventilator-associated event.

preventable fraction of these events and to evaluate the algorithm's clinical significance. ${ }^{3}$ VAEs have been shown to be associated with hospital mortality, prolonged mechanical ventilation, antimicrobial use, and ICU and hospital length of stays, and do show a stronger association with mortality than the original 2008 CDC VAP guidelines. ${ }^{4-8}$ Klompas points out in his 2017 analysis of the VAE algorithm that the NHSN's 2013 definitions provide an opportunity for hospitals to reassess prevention strategies. ${ }^{9}$ Multiple factors associated with the development of VAEs have been elucidated, including development of pneumonia, fluid overload, adult respiratory distress syndrome, and atelectasis. ${ }^{10}$ Strategies aimed toward preventing these conditions are well established in the critical care literature and have been compiled into defined best practice bundles readily used in ICUs worldwide. ${ }^{11} 12$

Nonetheless, it remains unclear whether or not VAEs are, in fact, preventable events. Among trauma patients, VAEs have been shown to be associated with mortality, but studies thus far mostly assess the infectious subset of VAEs, or IVACs. As Meagher et al note, the development of VAEs in the trauma population may be largely due to non-infectious causes such as inflammation associated with direct lung injury as a result of chest wall trauma or, alternatively, the sequelae of resuscitation and transfusion necessary to prevent early mortality from hemorrhagic shock. ${ }^{7314}$ Among our own trauma patients, we anecdotally observed that a proportion of VAEs were a result of an increase in PEEP from 5 to 8, and we questioned whether the VAE that followed was simply representative of the natural course after severe injury and resuscitation and its associated ventilator management, rather than a nosocomial complication of mechanical ventilation. Recognizing that the maximum PEEP infrequently surpassed $8 \mathrm{~cm} \mathrm{H}_{2} \mathrm{O}$ during the hospital course, we reasoned that we could eliminate these VAEs by simply starting out one measure higher $(6 \mathrm{~cm} \mathrm{H} 20)$ than our conventional starting point $(5 \mathrm{~cm} \mathrm{H} 20)$. In this study, we demonstrated that our VAE rate did indeed decrease with this practice change.

Much concern has been raised over the susceptibility of VAE surveillance and reporting to gaming. Minimizing the development of VAEs by redefining terms or misinterpreting data has been shown to be possible; as Klompas summarized in 2015, the CDC has had to clarify definitions of daily minimum
PEEP and $\mathrm{FiO}_{2}$ after Klein et al noted substantial discrepancies in VAE incidence depending on varying interpretations of these terms. ${ }^{10}{ }^{15}$ Variation in incidence has also been reported depending on whether electronic versus manual surveillance is used. ${ }^{16}$ Certainly, the PEEP and $\mathrm{FiO}_{2}$ modification criteria required for triggering a VAE are susceptible to gaming. Lilly et al demonstrated this by reducing VAEs by $93 \%$ by alternating the PEEP or $\mathrm{FiO}_{2}$ by 1 or 0.1 , respectively, on alternating days, thereby preventing their population from meeting VAE triggering requirements. ${ }^{17}$ Although ventilator management strategies may be developed for the sole purpose of evading VAE surveillance, it is important to note the triggers for VAE are relatively arbitrary. For example, a ventilator practice that includes optimization of PEEP via pulmonary compliance curves evaluated at regular intervals may result in repeated, small changes in PEEP that prevent that patient from meeting the VAE threshold as defined by the current algorithm. The intent of such a practice is not to game the system but to optimize oxygenation. Nonetheless, it would be likely that the rate of VAEs in this setting would be low.

With respect to our practice change, our ventilator management strategy was not borne from a desire to evade VAE surveillance. In fact, it originated from the recognition that many of our VAEs were simply a result of increasing PEEP to 8 in the routine ventilator management of trauma patients, and we questioned the clinical relevance of triggering a VAE in this specific scenario. Not only did an increase of initial PEEP from 5 to 6 decrease our incidence of VAEs by over $60 \%$ once adjusted by multivariate analysis, a significant association between VAE and mortality in the FY 2018 cohort was observed that was not apparent prior to the practice change. This observation supports our contention that many VAEs in our practice were, in fact, triggered by a PEEP change from 5 to 8 and that these particular VAEs were relatively immaterial to outcome. We anticipated that physicians would not be anchored to an initial PEEP increase interval of three. In fact, we anticipated that 8 would remain the target, given both a general physician comfort with that level of PEEP and a general human aversion to odd numbers (which is perhaps why, in our experience, PEEP is rarely if ever increased from 5 to 7 and so on). Although we did not specifically evaluate changes in PEEP intervals, we are comfortable, given the resultant decrease in VAE rate, that PEEP was not routinely increased from 6 to 9 .

There are several limitations to our study. It cannot be confirmed that every patient in the FY 2017 cohort and every patient in the FY 2018 cohort had initial PEEP settings of 5 and 6 respectively. Nonetheless, given the typical strict adherence to respiratory therapy guidelines on our trauma service, we are confident that nearly all of our patients had initial PEEP of 5 versus 6 according to cohort as presumed. Similarly, we are unable to account for any other differences in practice (in addition to the initial PEEP change) implemented across the two cohorts, but no other service guideline or practice changes

Table 4 Summary of logistic regression model predicting mortality

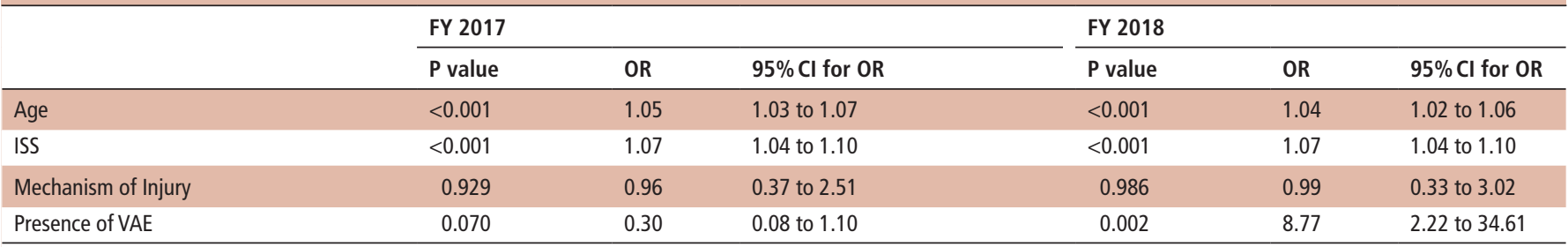

FY, fiscal year; ISS, Injury Severity Score; VAE, ventilator-associated event. 
were made over the study time period with respect to ventilator management, transfusion practice, or antibiotic stewardship. Nonetheless, ventilator management, although protocolized, is patient specific and changes made to ventilator modes, PEEP, and oxygen levels are dynamic and individualized to patient response. In addition, it is unknown whether or not the change from PEEP of 5 to 6 had any salutary or detrimental influence on pulmonary physiology. Nevertheless, we do not anticipate that a PEEP difference of $1 \mathrm{~cm} \mathrm{H}_{2} \mathrm{O}$ would have any measurable impact on pulmonary function. Notably, studies comparing much broader differentials in PEEP have demonstrated a lack of clinical impact. ${ }^{18} 19$ Lastly, relatively low numbers of patients in both cohorts make our results more prone to the effects of unconsidered confounders.

\section{CONCLUSION}

Mechanical ventilation can be both a life-sustaining measure and a necessary evil in critically ill trauma patients. The use of the CDC's algorithmic approach to VAEs as a quality indicator has been rigorously studied in the general critical care population. In trauma patient populations, however, it remains unclear whether VAEs are representative indicators of the quality of inpatient hospital care versus a reflection of the natural course of severe traumatic injury. Our study demonstrates a decreased incidence of reported VAEs in intubated trauma patients after a minor change in initial PEEP settings. In addition, we have demonstrated that excluding clinically insignificant VAEs as a result of our practice change results in our VAE cohort reflecting a better predictor of mortality. This reflection is in better alignment with original intent of the CDC's reporting criteria and allows us to focus our quality improvement processes targeted to a more clinically relevant patient population. At present, VAE is not a mandatory, public reporting requirement for hospitals. Nonetheless, we anticipate that this will not be the case in the near future, and our future may include public comparison of VAE rates among trauma centers. It is therefore worthwhile for trauma centers to consider how their ventilator management strategies may influence their rates of VAE, and whether or not VAE predicts mortality among their respective patients. A simple adjustment in initial PEEP may result in a lower rate of VAE. In addition, it may effectively make VAE a more clinically relevant metric in traumatically injured patient populations. Lastly, we encourage the NHSN to re-evaluate the VAE definition and consider revising the criterion regarding change in PEEP.

Contributors EF participated in the study design and interpretation. KMC participated in the data collection, analysis, and manuscript preparation, LGC participated in the study design and interpretation. JM participated in the data collection and interpretation. JAJ participated in the data collection and interpretation. AWM participated in the data collection and interpretation. JNB participated in the study design,interpretation, and critical review, HS-L participated in the study design, interpretation, and critical review. JAW participated in the study design,interpretation, and critical review.

Funding The authors have not declared a specific grant for this research from any funding agency in the public, commercial or not-for-profit sectors.

Competing interests None declared.

Patient consent for publication Not required

Ethics approval This study obtained approval from the St. Joseph's Hospital and Medical Center's internal Institutional Review Board. Approval number: PHXA-18500-235-73-21.

Provenance and peer review Not commissioned; externally peer reviewed.

Data availability statement All data relevant to the study are included in the article or uploaded as supplementary information.
Open access This is an open access article distributed in accordance with the Creative Commons Attribution Non Commercial (CC BY-NC 4.0) license, which permits others to distribute, remix, adapt, build upon this work non-commercially, and license their derivative works on different terms, provided the original work is properly cited, appropriate credit is given, any changes made indicated, and the use is non-commercial. See: http://creativecommons.org/licenses/by-nc/4.0/.

\section{ORCID iDs}

Kristina M Chapple http://orcid.org/0000-0001-5045-3524

Jessica A Johnson http://orcid.org/0000-0002-5342-9009

Andrew W Mezher http://orcid.org/0000-0002-9072-9510

James N Bogert http://orcid.org/0000-0002-6777-687X

\section{REFERENCES}

1 Klompas M. Interobserver variability in ventilator-associated pneumonia surveillance. Am J Infect Control 2010;38:237-9.

2 Centers for Disease Control and Prevention. Chapter 10: Ventilator-Associated Event (VAE). National healthcare safety network (NHSN) patient safety component manual, 2019. https://www.cdc.gov/nhsn/pdfs/pscmanual/pcsmanual current.pdf.

3 Magill SS, Li Q, Gross C, Dudeck M, Allen-Bridson K, Edwards JR. Incidence and characteristics of ventilator-associated events reported to the National healthcare safety network in 2014. Crit Care Med 2016;44:2154-62.

4 Boyer AF, Schoenberg N, Babcock H, McMullen KM, Micek ST, Kollef MH. A prospective evaluation of ventilator-associated conditions and infection-related ventilatorassociated conditions. Chest 2015;147:68-81.

5 Muscedere J, Sinuff T, Heyland DK, Dodek PM, Keenan SP, Wood G, Jiang X, Day AG, Laporta D, Klompas M, et al. The clinical impact and preventability of ventilatorassociated conditions in critically ill patients who are mechanically ventilated. Chest 2013;144:1453-60.

6 Zhu S, Cai L, Ma C, Zeng H, Guo H, Mao X, Zeng C, Li X, Zhao H, Liu Y, et al. The clinical impact of ventilator-associated events: a prospective multi-center surveillance study. Infect Control Hosp Epidemiol 2015;36:1388-95.

7 Meagher AD, Lind M, Senekjian L, Iwuchukwu C, Lynch JB, Cuschieri J, Robinson BRH. Ventilator-Associated events, not ventilator-associated pneumonia, is associated with higher mortality in trauma patients. J Trauma Acute Care Surg 2019;87:307-14.

8 Kobayashi H, Uchino S, Takinami M, Uezono S. The impact of ventilator-associated events in critically ill subjects with prolonged mechanical ventilation. Respir Care 2017:62:1379-86.

9 Klompas M. Ventilator-Associated events 5 years later. Respir Care 2017;62:1501-3.

10 Klompas M. Potential strategies to prevent ventilator-associated events. Am J Respir Crit Care Med 2015;192:1420-30.

11 Barnes-Daly MA, Phillips G, Ely EW. Improving Hospital survival and reducing brain dysfunction at seven California community hospitals: implementing pad guidelines via the ABCDEF bundle in 6,064 patients. Crit Care Med 2017;45:171-8.

12 Klompas M, Branson R, Eichenwald EC, Greene LR, Howell MD, Lee G, Magill SS, Maragakis LL, Priebe GP, Speck K, et al. Strategies to prevent ventilator-associated pneumonia in acute care hospitals: 2014 update. Infect Control Hosp Epidemiol 2014;35:915-36.

13 Younan D, Griffin R, Zaky A, Pittet J-F, Camins B. A comparison of outcomes of trauma patients with ventilator-associated events by diagnostic criteria set. Shock 2019:51:599-604.

14 Robinson BRH, Cohen MJ, Holcomb JB, Pritts TA, Gomaa D, Fox EE, Branson RD, Callcut RA, Cotton BA, Schreiber MA, et al. Risk factors for the development of acute respiratory distress syndrome following hemorrhage. Shock 2018:50:258-64.

15 Klein Klouwenberg PMC, van Mourik MSM, Ong DSY, Horn J, Schultz MJ, Cremer OL, Bonten MJM, MARS Consortium. Electronic implementation of a novel surveillance paradigm for ventilator-associated events. feasibility and validation. Am J Respir Crit Care Med 2014:189:947-55.

16 Mann T, Ellsworth J, Huda N, Neelakanta A, Chevalier T, Sims KL, Dhar S, Robinson ME, Kaye KS. Building and validating a computerized algorithm for surveillance of ventilator-associated events. Infect Control Hosp Epidemiol 2015;36:999-1003.

17 Lilly CM, Landry KE, Sood RN, Dunnington CH, Ellison RT, Bagley PH, Baker SP, Cody S, Irwin RS, UMass Memorial Critical Care Operations Group. Prevalence and test characteristics of national health safety network ventilator-associated events. Crit Care Med 2014:42:2019-28.

18 Brower RG, Lanken PN, Maclntyre N, Matthay MA, Morris A, Ancukiewicz M, Schoenfeld D, Thompson BT, National Heart, Lung, and Blood Institute ARDS Clinical Trials Network. Higher versus lower positive end-expiratory pressures in patients with the acute respiratory distress syndrome. N Engl J Med 2004;351:327-36

19 Meade MO, Cook DJ, Guyatt GH, Slutsky AS, Arabi YM, Cooper DJ, Davies AR, Hand $L E$, Zhou $Q$, Thabane $L$, et al. Ventilation strategy using low tidal volumes, recruitment maneuvers, and high positive end-expiratory pressure for acute lung injury and acute respiratory distress syndrome: a randomized controlled trial. JAMA 2008;299:637-45. 